\title{
Resolving Runaway Electron Distributions in Space, Time, and Energy
}

\author{
C. Paz-Soldan, ${ }^{1}$ C. M. Cooper, ${ }^{2}$ P. Aleynikov ${ }^{3}$ N. W. Eidietis,${ }^{1}$ A. Lvovskiy, ${ }^{2}$ D. \\ C. Pace, ${ }^{1}$ D. P. Brennan, ${ }^{4}$ E. M. Hollmann, ${ }^{5}$ C. Liu, ${ }^{4}$ R. A. Moyer ${ }^{5}$ and D. Shiraki ${ }^{6}$ \\ ${ }^{1}$ General Atomics, San Diego, CA, USA* \\ ${ }^{2}$ Oak Ridge Associated Universities, Oak Ridge, TN, USA \\ ${ }^{3}$ Max-Planck Institute for Plasma Physics, Greifswald, Germany \\ ${ }^{4}$ Princeton University, Princeton, NJ, USA \\ ${ }^{5}$ University of California San Diego, La Jolla, CA, USA \\ ${ }^{6}$ Oak Ridge National Laboratory, Oak Ridge, TN, USA
}

(Dated: January 30, 2018)

\begin{abstract}
Areas of agreement and disagreement with present-day models of $\mathrm{RE}$ evolution are revealed by measuring MeV-level bremsstrahlung radiation from runaway electrons (REs) with a pinhole camera. Spatially-resolved measurements localize the RE beam, reveal energy-dependent RE transport, and can be used to perform full two-dimensional (energy and pitch-angle) inversions of the RE phasespace distribution. Energy-resolved measurements find qualitative agreement with modeling on the role of collisional and synchrotron damping in modifying the RE distribution shape. Measurements are consistent with predictions of phase-space attractors that accumulate REs, with non-monotonic features observed in the distribution. Temporally-resolved measurements find qualitative agreement with modeling on the impact of collisional and synchrotron damping in varying the RE growth and decay rate. Anomalous RE loss is observed and found to be largest at low energy. Possible roles for kinetic instability or spatial transport to resolve these anomalies are discussed.
\end{abstract}

\section{INTRODUCTION}

Due to the severe potential for damage to the reactor walls, runaway electrons (REs) pose a significant operational risk to fusion-grade tokamaks such as ITER [1]4. RE populations are accelerated to very high energy (tens of $\mathrm{MeV}$ ) by the large electric fields induced during a tokamak disruption. The first line of defense against REs is to avoid their generation by either avoiding disruptions entirely, or by developing disruption mitigation strategies that prevent the formation of runaway electrons. A second line of defense is to manipulate the post-disruption RE beam to dissipate its energy prior to contact of the $\mathrm{RE}$ beam with the material surface. An example action is the injection of a large quantity of high- $\mathrm{Z}$ nobel gas, either in frozen or gaseous form.

Empirical tuning of RE control actuators in future tokamaks will be limited due to the potential for damage. Instead, a predictive understanding is needed to select and optimize actuators for RE control. Presentday experiments have a crucial role to play in validating theoretical predictions of RE dissipation. This role is most direct in the second line of defense, where similar conditions to what is expected in ITER can be accessed in existing tokamaks.

Theory and modeling work to understand the dissipation of RE populations is actively pursued. Early models taking into account small angle Coulomb collisions [5] and later large angle collisions [6] identified the crucial role of the 'critical electric field' $\left(E_{\mathrm{C}}\right)$, which defines the electric field at which collisional drag balances electric field acceleration in the relativistic limit. Later work

\footnotetext{
* paz-soldan@fusion.gat.com
}

identified the potential for synchrotron damping to elevate the effective $E_{\mathrm{C}}$ [7, 8, and more recently analytic treatments [9] and computational methods [10 16] were developed to combine the effects of synchrotron damping and pitch-angle scattering off high- $\mathrm{Z}$ ions. These works confirmed that synchrotron and pitch-angle scattering can together elevate the effective $E_{\mathrm{C}}$ above early model predictions.

Several experiments concurrently yielded evidence of very elevated $E_{\mathrm{C}}$ values above both the early model prediction and the elevated $E_{\mathrm{C}}$ from more modern models [17-19, both using trace-level REs naturally occurring in low density Ohmic plasmas as well as in post-disruption 'plateau' RE beams [20, 21]. More recently, diagnostic improvements enabled new experimental resolution in both space and time to measure the RE distribution function, yielding improved comparison to modeling [22]. This work found that while most qualitative trends were captured, quantitative discrepancies remained. Most notably, theory did not yet match the experimentally observed very elevated $E_{\mathrm{C}}$.

In this paper precise measurements of $\mathrm{RE}$ distribution functions and dissipation rates in the spatial, temporal, and energy domains are presented, enabled by a new RE diagnostic called the 'Gamma Ray Imager' (GRI) 23, 24 deployed on the DIII-D tokamak. Direct comparisons to modeling are made wherever possible, highlighting areas of agreement and disagreement. This paper expands on measurements first presented in Ref. 22 by 1 ) describing experimental methodology and model comparisons in greater detail, 2) describing expected spatial effects using a synthetic diagnostic, 3) presenting supporting measurements from additional diagnostics, 4) and discussing candidate mechanisms to explain the observed discrepancies. 
The remainder of this paper is structured as follows. Section II describes the measurement technique and plasma scenario employed in this work. Section III describes measurements in the spatial domain and how radial and angular RE profiles are resolved. Section [IV describes the inversion of RE energy distributions from localized hard X-ray (HXR) emission and subsequent comparison of $\mathrm{RE}$ distribution shapes against theoretical predictions as dissipation terms are varied. Section $\mathrm{V}$ describes time-domain measurements of RE emission growth and decay, including parametric dependencies and comparisons to theoretical predictions. Finally, a summary of observations along with areas for further improvements in modeling and measurement are provided in Sec. VI.

\section{DESCRIPTION OF DIAGNOSTIC, PLASMA SCENARIO, AND MODELING APPROACH}

\section{A. Diagnostic Sensitivity}

This work focuses on measurements of the RE population made by the GRI system installed on DIII-D [23, 24]. The GRI is a tangentially-viewing pinhole camera made of lead, with collimation provided along 123 discrete sight-lines as shown in Fig. 11(a). When a RE scatters off a plasma nuclei, HXR emission is generated via the bremsstrahlung mechanism. If the HXR is emitted along a sight-line of the GRI, the HXR will enter the pinhole and be registered on a pulse-height counting detector consisting of an integrated Bismuth-Germanate (BGO) scintillating crystal, a photo-diode, and pre-amplification electronics. The output of each detector is digitized at 10 $\mathrm{MHz}$ sample rate, enabling individual pulses to be discriminated, as shown in Fig. 1(b). The height of the pulse corresponds linearly to the energy of the measured $\operatorname{HXR}\left(E_{\gamma}\right)$. Each HXR pulse is assigned an energy and a time-stamp, and histograms are later formed to build up time-resolved distributions of the RE HXR emission $\left(f_{\gamma}\right)$ along each sight-line. As will be described in Sec. [IV] the HXR energy distribution $\left(f_{\gamma}\right)$ in turn carries information about the incident $\mathrm{RE}$ distribution $\left(f_{e}\right)$, which can be inverted with some assumptions. Each sight-line views a different part of the plasma cross-section, as shown in Fig. 11(c). Along each sight-line, different angles are made between the equilibrium magnetic field direction ( $\hat{b}$, obtained from equilibrium reconstructions) and the sight-line orientation $(\hat{k})$. The minimum $\theta_{\hat{k} \hat{b}}$ along the sight-line is used to color-code the view. The $\theta_{\hat{k} \hat{b}}$ parameter is crucial to understand the spatial dependence of the emission, as will be described in Sec. III Note that of the 123 available sight-lines, approximately 20 were populated for these first experiments. Further details of the diagnostic are provided in Refs. 23 and 24 .

Beyond the GRI, measurements of the RE population are also made with diagnostics sensitive to bremsstrahlung and synchrotron radiation from distinct parts of RE phase space, as summarized in Fig. 2 DIII$\mathrm{D}$ is equipped with several scintillating HXR detectors placed several meters from the tokamak, here called the 'distant HXR' detectors. These detectors have no collimation and thus sample the entire distribution with uncertain weighting. This sort of diagnostic was previously used to identify elevated thresholds for the transition from RE growth to decay [18, 19]. Synchrotron emission (SE) is measured in the visible range by a tangentially viewing camera system, capable of generating detailed spatial images of the RE emission intensity pattern. These images have been reported in several experimental publications [25, 27] and only recently has the SE shape been predicted by simulation [28, 29]. Sensitivity scans in simulation indicate that the visible SE originates from quite energetic REs of medium pitch-angle. Finally, electron cyclotron emission (ECE) radiometers at high frequency (here from 140-300 GHz) are preferentially sensitive to non-thermal synchrotron emission from REs. Due to the radial viewing geometry of the ECE radiometers on DIII-D, these diagnostics probe the high pitch-angle RE population. Simulation further indicates that for these frequencies the energy of the RE responsible for the emission is low [15. While this work focuses on the GRI system, measurements from these other diagnostics are also presented to corroborate GRI observations.

\section{B. Scenario Description}

This work reports measurements made in low density Ohmic plasmas during flat-top. Low density operation free from error field penetration supports the excitation of trace-level RE populations through the 'Dreicer' mechanism [30, which as time goes on begin to multiply through the secondary ('Avalanche') mechanism. A typical discharge evolution is shown in Fig. 3 with the early period with strong primary and secondary generation termed the growth phase. Typical parameters in the growth phase are electron density $\left(n_{e}\right)$ of $0.5 \times 10^{19}$ $\mathrm{m}^{-3}$, electron temperature $\left(T_{e}\right)$ of $1.8 \mathrm{keV}$, toroidal field $\left(B_{T}\right)$ of $1.4 \mathrm{~T}$, plasma current $\left(I_{P}\right)$ of $0.8 \mathrm{MA}$, and loop voltage $\left(V_{\text {loop }}\right)$ of $0.6 \mathrm{~V}$.18, A typical avalanche multiplication time in these conditions is $0.5 \mathrm{~s}$, thus several multiplications occur during the growth phase. During this time the distant HXR signal increases several-fold exponentially [Fig. 3(b)].

After several seconds the discharge transitions to the 'dissipation phase', where plasma parameters are changed to observe their effect on the RE distribution. As the dissipation phase usually involves gas puffing to raise $n_{e}$, the transition time is denoted $t_{\text {puff. }}$. While previously $t_{\text {puff }}$ occurred at a fixed time, the DIII-D plasma control system was upgraded to allow asynchronous control based on the magnitude of high-harmonic ECE at $140 \mathrm{GHz}$, a frequency where the ECE is dominated by non-thermal emission. As seen in Fig. 3(c), when the 

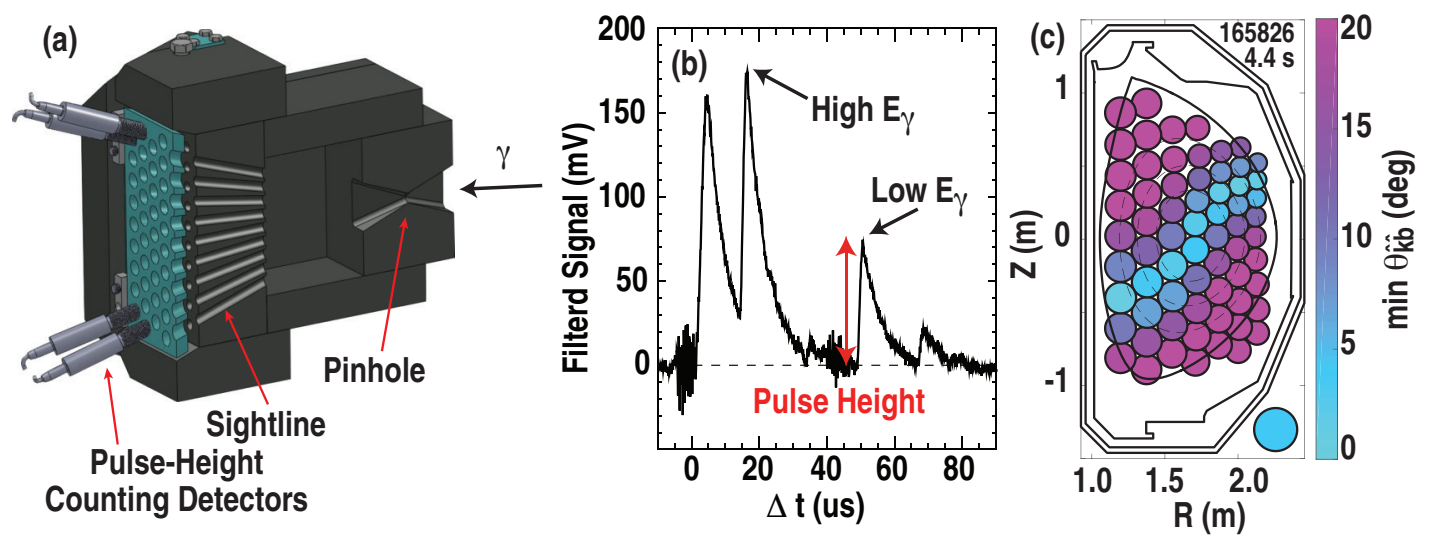

FIG. 1. (a) GRI pinhole camera geometry, (b) raw digitized signal from a single detector, and (c) sight-lines into the plasma at the tangency plane. Colors indicate the minimum angle $\left(\theta_{\hat{k} \hat{b}}\right)$ between the magnetic field orientation $(\hat{b})$ and the sight-line orientation $(\hat{k})$.
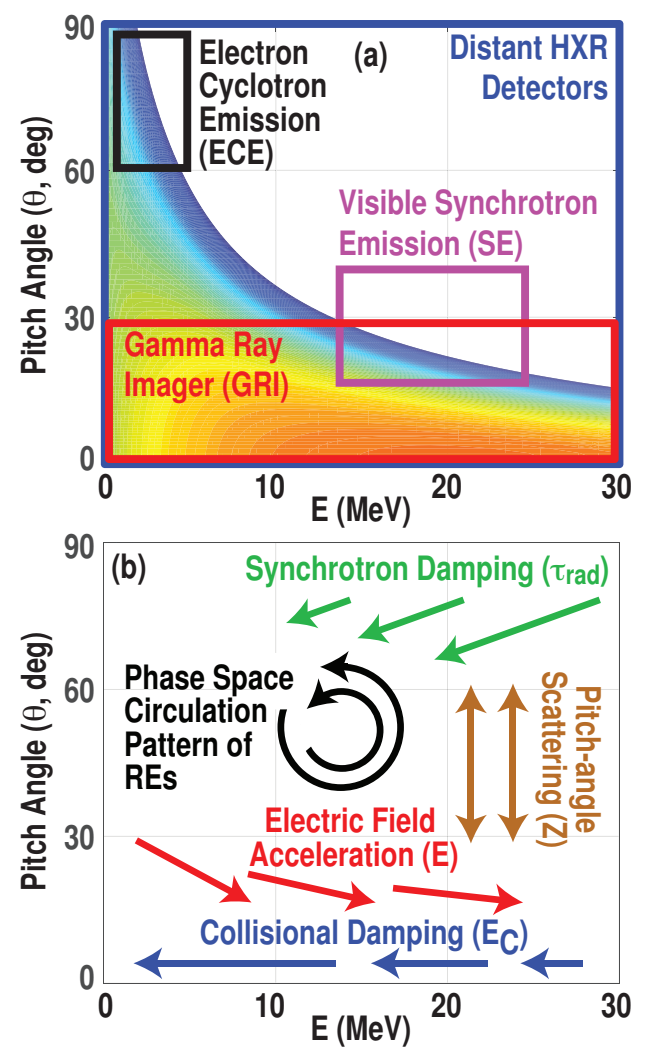

FIG. 2. (a) Region of phase space responsible for emission measured by the various diagnostics used in these experiments, together with an example predicted $f_{e}$ (color contours) to indicate where REs are expected in phase space. (b) Cartoon description of influence of electric field acceleration, collisional damping, pitch-angle scattering, and synchrotron damping on REs in phase space.

ECE emission exceeds a pre-set trip level the dissipation phase is entered. Maintaining a constant trip level enables roughly the same RE distribution to be achieved in every pulse prior to entering the dissipation phase. If the trip is not enabled, the rapid rise in ECE progresses and the discharge evolves to tearing instability as described in Ref. 31.
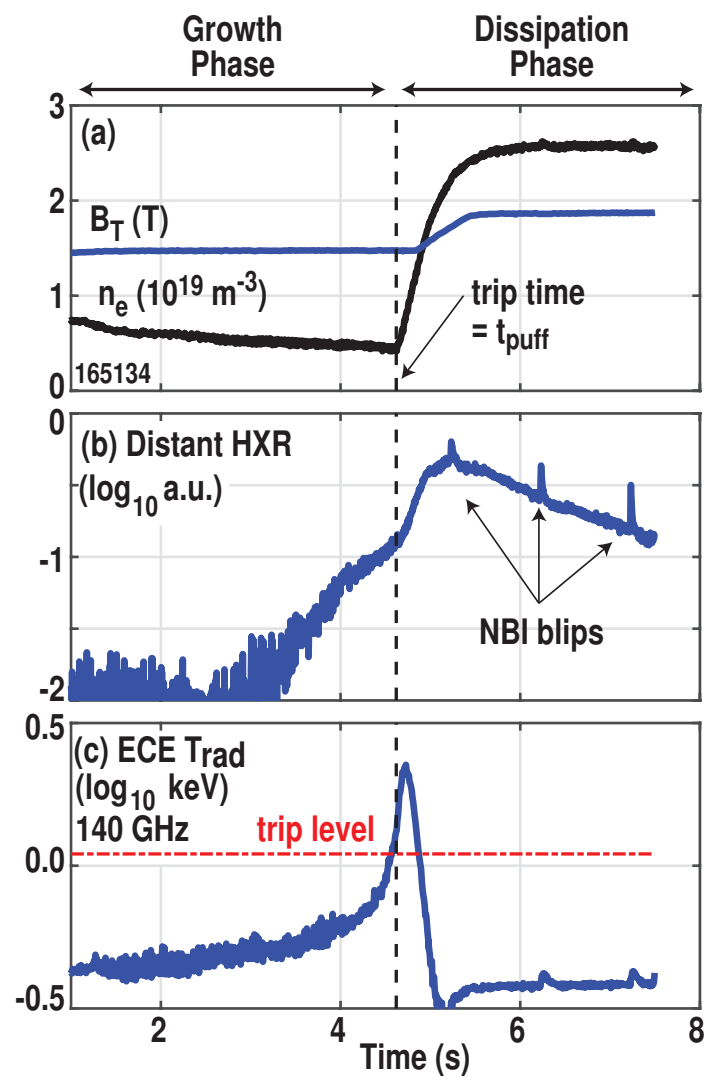

FIG. 3. Evolution of (a) $n_{e}$ and $B_{T}$, (b) distant HXR detector, and (c) ECE radiometer illustrating the asynchronous transition from a RE growth to RE dissipation phase when a critical level of ECE emission is reached. Experiments discussed herein are conducted by varying the plasma parameters in only the dissipation phase.

\section{Modeling Approach}

In order to predict expected RE distribution functions $\left(f_{e}\right)$ and growth or decay rates, the time-evolution of nondimensional parameters determining the $\mathrm{RE}$ distribution must be known. In contrast to the complex and rapid dynamics of a disruptive plasma, both the growth and 
decay phase of the Ohmic plasma are quasi-stationary and all relevant parameters are measured with sufficient time-resolution. This generates uniquely confident input parameters to modeling. The modeling inputs include the ratio of the imposed electric field $E$ to the critical electric field $E_{\mathrm{C}}$, defined as

$$
\frac{E}{E_{\mathrm{C}}} \equiv \frac{4 \pi \varepsilon_{0}^{2} m_{e} V_{C}^{2}}{n_{e} e^{3} \ln \Lambda} \approx 10 \frac{V_{\text {loop }}[\mathrm{V}]}{n_{e}\left[10^{19} \mathrm{~m}^{-3}\right]},
$$

and the ratio of the collision time to the synchrotron damping time $\hat{\tau}_{\text {rad }}$, defined as

$$
\hat{\tau}_{\mathrm{rad}} \equiv \frac{3}{2}\left(\frac{m_{e} \ln \Lambda}{\epsilon_{0}}\right) \frac{n_{\mathrm{e}}}{B_{T}^{2}} \approx 28 \frac{n_{e}\left[10^{19} \mathrm{~m}^{-3}\right]}{\left(B_{T}[\mathrm{~T}]\right)^{2}} .
$$

In addition, the effective charge $Z$, important for pitch-angle scattering, is controlled by deliberate injection of a nitrogen impurity species and is measured using charge-exchange spectroscopy of both nitrogen and ambient carbon populations. Finally, the electron temperature $T_{e}$, important for Dreicer generation, is measured with Thomson scattering. The impact of these different effects (electric field acceleration, collisional damping, pitch-angle scattering, and synchrotron damping) in RE phase space is shown in cartoon format in Fig 2(b). With all effects acting together, circulation patterns in RE phase space can be formed.

To model the evolution of $f_{e}$ the time-dependent relativistic 2-D Fokker-Planck equation (such as Ref. [32]) is solved numerically inputting measured on-axis (spatially 0 -D) plasma parameters of $E / E_{\mathrm{C}}, \hat{\tau}_{\text {rad }}$, and $Z$. The equation as in Ref. 9] is solved with two amendments. First, the collision operator is extended to be valid for lower energies using an approach similar to Refs. 33, 34. Second, an approximate secondary source is included which captures the effect of a finite energy incident electron population. This treatment accurately captures the analytical results of RE generation models [5, 6, 35] as well as the near-threshold regime [9]. This process generates a complete two-dimensional $f_{e}$, which is then integrated from pitch-angle of 0 to $30^{\circ}$, corresponding to the approximate angular coverage of the GRI system as will be described in Sec. III.

\section{Relevance to ITER Post-Disruption Conditions}

Owing to the wide variety of plasma parameters $\left(n_{e}, B_{T}, V_{\text {loop }}\right)$ accessible in the dissipation phase, nondimensional parameters $E / E_{\mathrm{C}}, \hat{\tau}_{\text {rad }}$, and $Z$ can be made surprisingly similar to those expected in ITER postdisruptive conditions. For example, owing to the low $B_{T}$ of these plasmas (1.4 T) as compared to ITER (5.3 T), to match the expected value of $\hat{\tau}_{\text {rad }}(\approx 70)$ during a postdisruption ITER RE beam requires very low density operation in DIII-D. In terms of $Z$, impurity injection in the
Ohmic plasmas has raised $Z$ to about 4 , similar to the expectation of $Z \approx 5$ in ITER for partially-assimilated neon secondary injection. A wide range of $E / E_{\mathrm{C}}$ is expected during a single discharge in ITER, ranging from $1-2$ to several hundred [36, 37], whereas in these plasmas $E / E_{\mathrm{C}}$ is well controlled between $\approx 2-8$. It should be noted significant uncertainty exists in the parameters expected for ITER, largely owing to uncertainties in the degree of impurity assimilation. Thus, these experiments are not meant to match any specific prediction for ITER, but rather explore effects in a parametric space that should overlap with eventual ITER values.

Notwithstanding the surprisingly good nondimensional match of $\left(E / E_{\mathrm{C}}, \hat{\tau}_{\text {rad }}\right.$, and $\left.Z\right)$ to expected ITER conditions, the low density scenario is not expected to yield ITER-relevant results for some aspects of the RE problem. For example, as these plasmas are fully ionized, they cannot be used to validate predictions of enhanced dissipation due to partial screening 38, 39], nor would they be well suited to understand phenomenology associated with the RE final loss 40. Impurity transport into a low density Ohmic plasma with REs is also likely not a valid analog to the post-disruption condition [4].

\section{RESOLVING THE SPATIAL DOMAIN: RADIAL AND PITCH-ANGLE RE PROFILES}

Each collimated sight-line of the GRI system views a different portion of the plasma cross section, as shown in Fig. 1(c). By comparing the emission recorded along each sight-line, information about the spatial and angular distribution of the RE population can be inferred. However, in order to interpret these measurements the expected diagnostic sensitivity to spatially uniform and mono-energetic RE populations must first be understood. The expected sensitivity is computed using the synthetic diagnostic introduced in Sec. II A, with a complete description given in Ref. 24. This section will explore synthetic diagnostic dependencies alongside a comparison to experimental data.

The synthetic diagnostic is used to predict the HXR emission shape from a uniform RE density $\left(n_{\mathrm{RE}}\right)$ beam of a certain minor radius $r_{\text {beam }}$. This beam is given a single electron energy $\left(E_{e}\right)$, and zero pitch-angle. The expected emission as a function of $E_{\gamma}$ is then computed for all of the sight-lines that intersect the plasma. As shown in Fig. 4, $r_{\text {beam }}$ increases the size of the emission spot, while increasing $E_{e}$ increases the sharpness along the line of low $\theta_{\hat{k} \hat{b}}$.

This study identifies two directions most useful for reduced analysis. First, for analysis of the radial profile, it is useful to focus on the line of low $\theta_{\hat{k} \hat{b}}$ as this deemphasizes changes due to $E_{e}$ variations. Second, for analysis of the phase space profile, important information can be gained by comparing emission from different sight-lines sampling the same flux surface. These direc- 

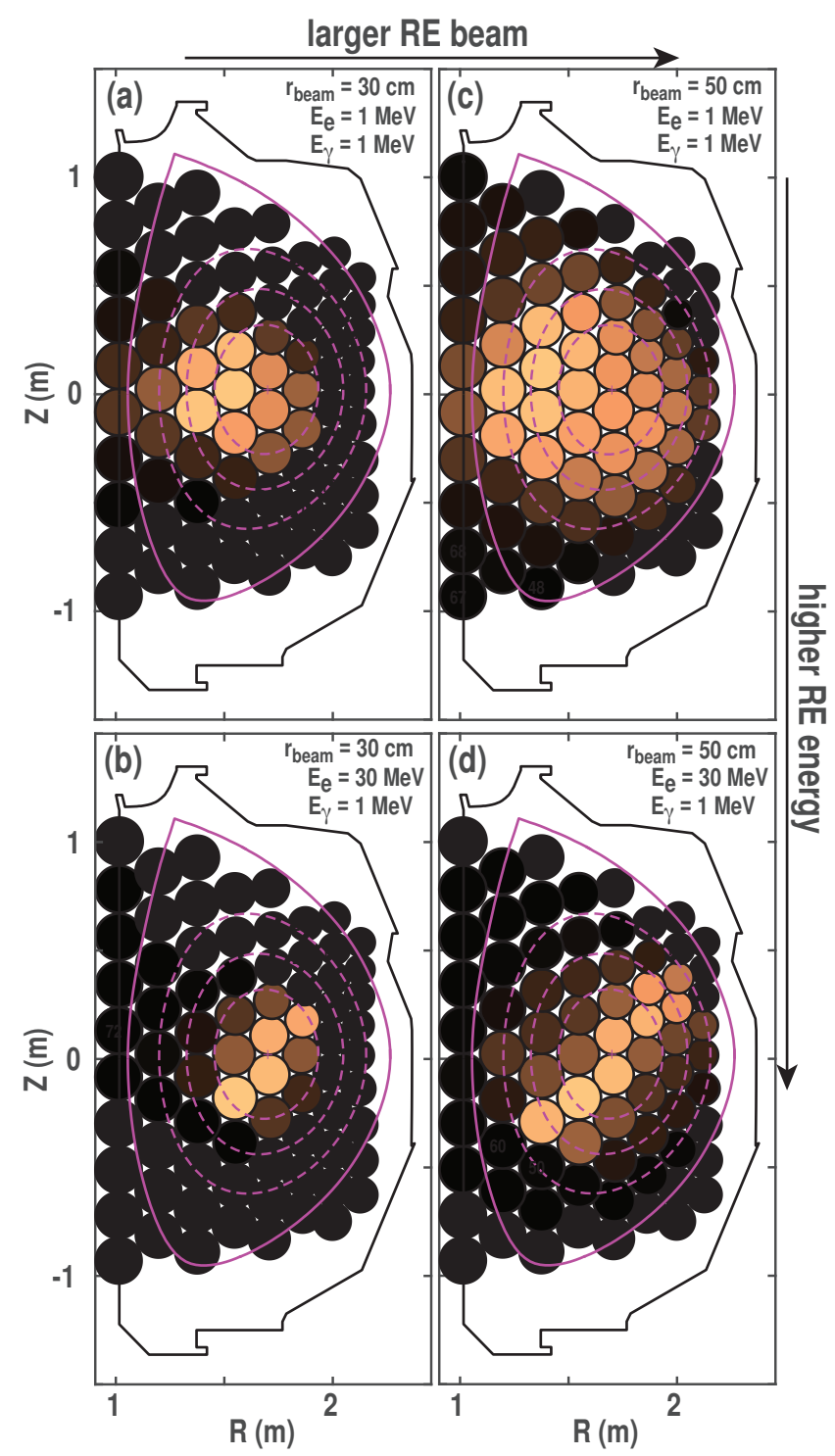

FIG. 4. Synthetic diagnostic prediction of HXR emission intensity pattern at a single $E_{\gamma}$ as a function of $r_{\text {beam }}$ and $E_{e}$, assuming zero pitch-angle. Increasing $r_{\text {beam }}$ increases the size of the emission spot, while increasing $E_{e}$ increases the sharpness along the line of low $\theta_{\hat{k} \hat{b}}$.

tions will now be considered separately in Sections III A and III B.

Note that the bremsstrahlung rate coefficients indicate that while the shape of the emission is very sensitive to $E_{e}$, there is no significant spatial variation with $E_{\gamma}$ for constant $E_{e}$. Thus, for all model-produced images $E_{\gamma}=1$ $\mathrm{MeV}$ is used. Note also that the synthetic diagnostic at present uses a zero-pitch angle approximation, which future work will relax.

\section{A. Radial Profile Resolution}

Synthetic diagnostic predictions along the low $\theta_{\hat{k} \hat{b}}$ line are examined as a function of $E_{e}$ and $r_{\text {beam. }}$. As seen in
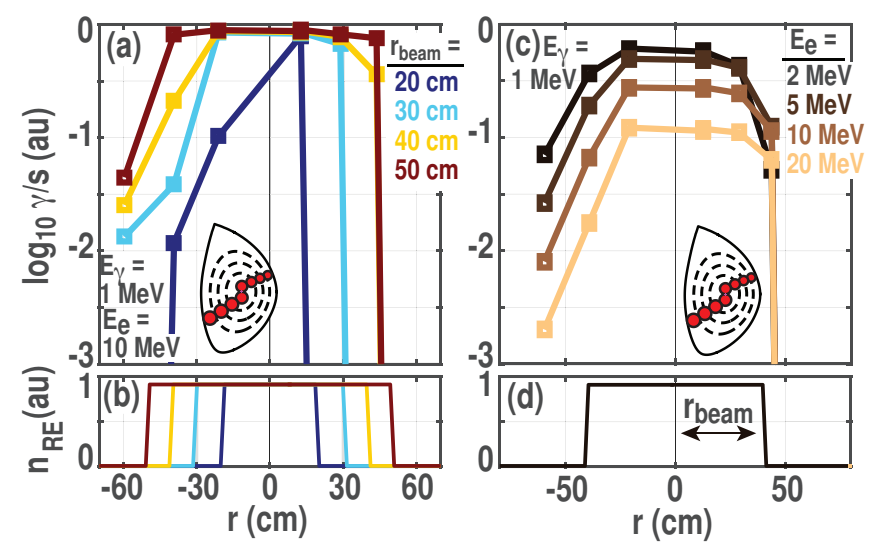

FIG. 5. Synthetic diagnostic predictions of the dependence of emission along the low $\theta_{\hat{k} \hat{b}}$ line to (a-b) $r_{\text {beam }}$ and (c-d) $E_{e}$. The inset indicates the sight-lines considered.

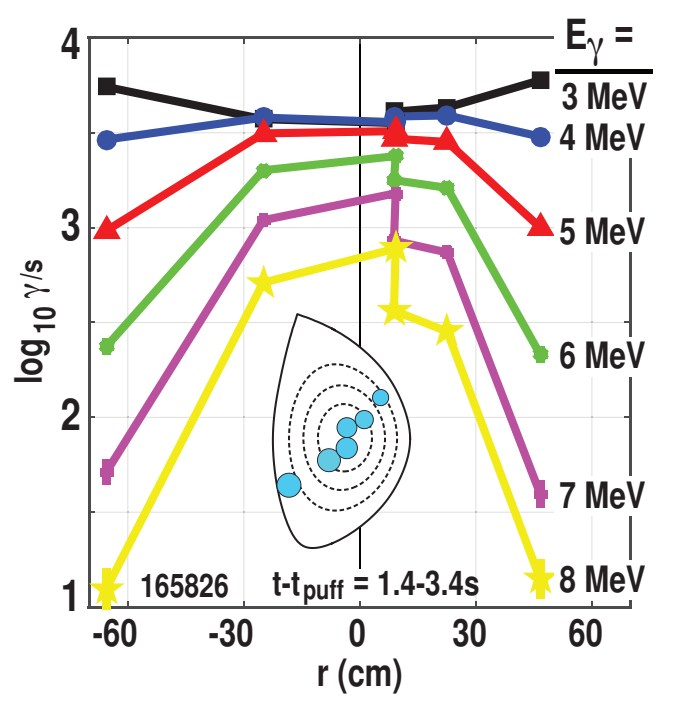

FIG. 6. Experimental measurement of the emission dependence along the low $\theta_{\hat{k} \hat{b}}$ line as a function of $E_{\gamma}$, indicating a convolution of the $r_{\text {beam }}$ and $E_{e}$ effects isolated in Fig. 5

Fig. 4. a strong sensitivity of the emission along the low $\theta_{\hat{k} \hat{b}}$ line is expected with $r_{\text {beam }}$. Focusing on the low $\theta_{\hat{k} \hat{b}}$ line, the dominant effect of $r_{\text {beam }}$ is seen in Fig. 5(a). Larger and smaller $r_{\text {beam }}$ map directly onto larger and smaller emission patterns. The break in the emission is especially sharp along the low-field side (LFS), while on the high-field side (HFS) it is more gradual. That the emission is asymmetric from LFS to HFS is reminiscent of the synchrotron emission images [ex. Fig. 12(f)].

The effect of $E_{e}$ along the low $\theta_{\hat{k} \hat{b}}$ line is more muted, as can be seen in Fig. 5(c). As $E_{e}$ is increased, a larger decrease is found on the HFS than on the LFS. This supports the interpretation that the increased signal on the HFS is simply an instrumental effect resulting from the longer integration lengths along inboard sight-lines, since at higher $E_{e}$ the emission is more localized to the tangency plane as opposed to coming from the entire sightline [24. Further LFS/HFS asymmetries may arise from untreated finite pitch-angle effects. 


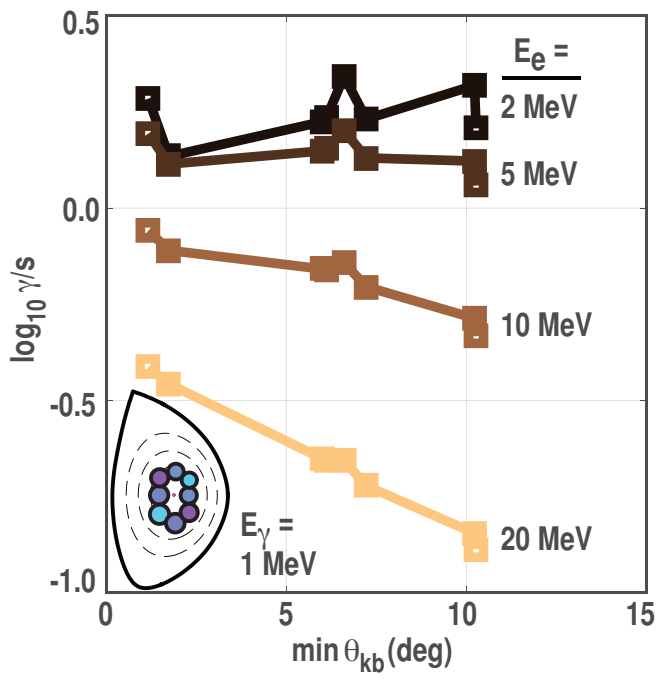

FIG. 7. Synthetic diagnostic prediction of emission variations between sight-lines viewing the same flux surface as $E_{e}$ is varied with fixed $n_{\mathrm{RE}}$ and $r_{\text {beam }}$, illustrating increased sharpness to low $\theta_{\hat{k} \hat{b}}$ at high $E_{e}$. Data is plotted against the minimum $\theta_{\hat{k} \hat{b}}$ of the sight-line.

Since the RE current is predominantly carried by few$\mathrm{MeV}$ electrons [3], these measurements can be used to measure the size of the $\mathrm{RE}$ current channel. This is an advantage over conventional visible SE images, since these images are dominated by the few highest energy electrons $(\geq 15 \mathrm{MeV}[28,29])$ and the current carried by these electrons is vanishingly small.

Experimental measurements along the low $\theta_{\hat{k} \hat{b}}$ line, shown in Fig. 6 indicate a strong variation of the RE beam size with $E_{\gamma}$ and thus $E_{e}$, and appear to have a convolution of the $E_{e}$ and $r_{\text {beam }}$ effects isolated in Fig. 5. This indicates that energy-dependent transport mechanisms are acting, although their origin is not yet understood. Measurements are also consistent with an asymmetry from LFS to HFS.

These radial measurements constitute a new frontier for RE model validation, as the vast majority of existing models do not include spatial effects, sacrificing spatial degrees of freedom to focus on phase space effects. Where spatial variations are treated, it is usually with an ad-hoc diffusion coefficient for the RE population [42]. These measurements can serve to empirically measure diffusion coefficients and understand their parametric dependencies.

\section{B. Angular Profile Resolution}

Synthetic diagnostic dependencies along a given flux surface are found to be most strongly sensitive to $E_{e}$, shown in Fig. 7 and better representing the increased 'sharpness' of the emission pattern in Fig. 4. The simplification of zero pitch-angle used, however, has direct implications for this spatial direction. This is because in the limit of high $E_{e}$, forward beamed emission would

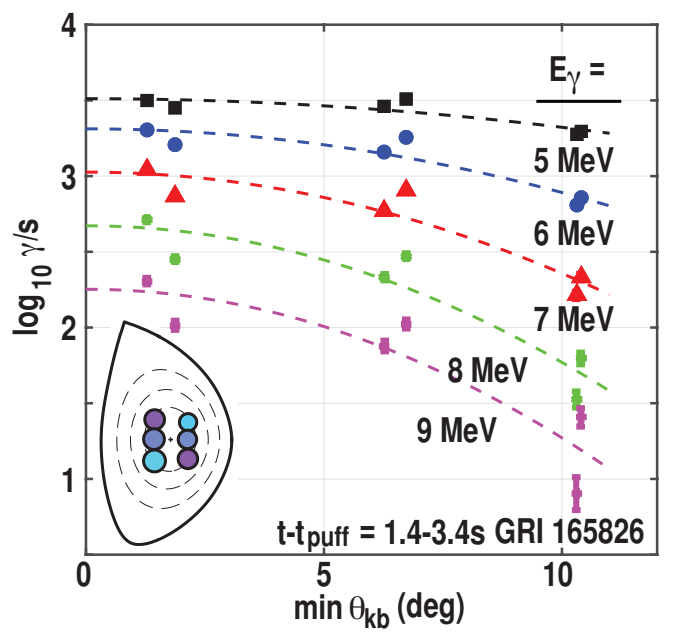

FIG. 8. Experimentally measured emission along the same sight-lines, with significantly more emission measured at low $\theta_{\hat{k} \hat{b}}$.

still reach a sight-line with high $\theta_{\hat{k} \hat{b}}$ if the electron pitchangle matches $\theta_{\hat{k} \hat{b}}$. Thus, emission profiles along the same flux surface contain information on the pitch-angle distribution of the RE population, which future work will examine.

The importance of pitch-angle can be seen by considering experimental measurements along these same slightlines, shown in Fig. 8. Significant variations are seen with $E_{\gamma}$ from 5-9 MeV. This is greater than the variation expected from increasing $E_{e}$ from 5 to $9 \mathrm{MeV}$ at constant $n_{\mathrm{RE}}$ (shown in Fig. 7), and thus indicates the underlying $n_{\mathrm{RE}}$ is varying with $E_{e}$. Further, the increased localization to low $\theta_{\hat{k} \hat{b}}$ of the $E_{\gamma}=9 \mathrm{MeV}$ emission indicates some of this emission is be due to electrons with $E_{e}>9$ $\mathrm{MeV}$. These measurements point to the future possibility of full two-dimensional inversions of the $\mathrm{RE}$ distribution function, with energy information provided by $f_{\gamma}$ and angular information provided by comparison of views of the same flux surface and different $\theta_{\hat{k} \hat{b}}$.

To conclude, resolution in the spatial domain is provided by comparing emission along the different collimated views. A synthetic diagnostic allows assessment of expected emission patterns from RE beams of constant $n_{\mathrm{RE}}$ and different $r_{\text {beam }}$ and $E_{e}$ (assuming zero pitchangle). A radial profile can be formed by looking along the line of low $\theta_{\hat{k} \hat{b}}$, and experimental data finds different increasing spatial gradients with increasing $E_{\gamma}$. Angular profiles can be generated by comparing views of the same flux surface with different $\theta_{\hat{k} \hat{b}}$, pointing to future energy and pitch-angle resolved $f_{e}$ inversions.

\section{RESOLVING THE ENERGY DOMAIN: RE DISTRIBUTION FUNCTIONS}

This section will describe how the energy spectrum $\left(f_{\gamma}\right)$ of incoming HXR pulses can be converted into 1-D RE energy distribution functions $\left(f_{e}\right)$. The dependence 

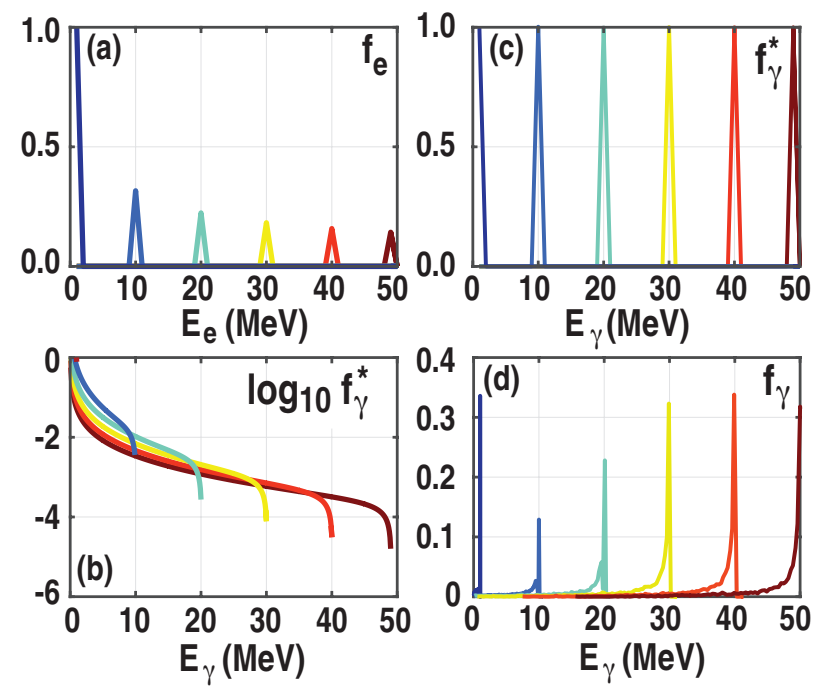

FIG. 9. Convolutions used in $f_{e}$ inversion. (a) A monoenergetic $f_{e}$ translates to (b) a spectrum of HXRs $\left(f_{\gamma}^{*}\right)$ from bremsstrahlung. (c) A mono-energetic $f_{\gamma}^{*}$ then undergoes (d) Compton scattering inside the scintillating crystal spreading $f_{\gamma}^{*}$ into the final measured $f_{\gamma}$.

of the inverted $f_{e}$ on experimental actuators will then be described alongside comparison to modeling.

\section{A. Inversion Technique}

The raw experimental measurement of the GRI system is a spectrum of HXR energies from individual HXR scintillation events. To go from this spectrum $\left(f_{\gamma}\right)$ to the RE distribution function $\left(f_{e}\right)$, two convolutions are needed - first the conversion from $f_{e}$ in the plasma to the incident HXR distribution entering the detector volume. This intermediate distribution is termed $f_{\gamma}^{*}$. The second convolution takes $f_{\gamma}^{*}$ to the final HXR distribution (termed $f_{\gamma}$ ) measured by scintillation light exiting each detector crystal.

The first convolution relies on computation of bremsstrahlung rate coefficients [43] together with sightline geometric information. The same synthetic diagnostic tool used in Sec. III is again used to calculate the HXR energy spectrum $\left(f_{\gamma}\right)$ from a series of monoenergetic $E_{e}$. Figure $9(\mathrm{~b})$ shows the results of this convolution for a series of mono-energetic $f_{e}$ spaced $10 \mathrm{MeV}$ apart [Fig. 9(a)] for a central viewing sight-line. Experimental inversions typically use spectra from monoenergetic $f_{e}$ spaced $1 \mathrm{MeV}$ apart.

The second convolution relies on knowledge of the scintillating crystal geometry and material properties to calculate the expected Compton scattering within the crystal using Monte-carlo methods. This technique is used to compute the registered $E_{\gamma}$ on the detector from a monoenergetic beam of incident HXRs [44. The results of these calculations for the GRI BGO scintillating crystals are shown in Fig. 9(c)-(d) for an input mono-energetic $f_{\gamma}^{*}$.

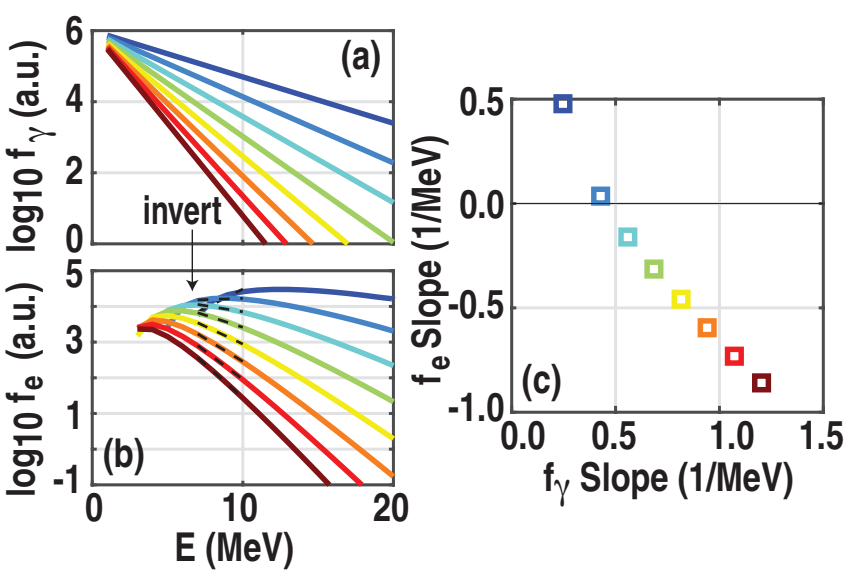

FIG. 10. Inversion results for (a) a family of $f_{\gamma}$ with different slopes, which (b) map to either peaked, flat, or inverted $f_{e}$. (c) Summarizes the relationship between $f_{e}$ and $f_{\gamma}$ slope.

These two convolutions are performed together to compute the expected $f_{\gamma}$ from mono-energetic $f_{e}$. The inversion process can then proceed using this information together with an onion-peel method, taking a key assumption: the maximum $E_{\gamma}$ recorded is taken to be the maximum $E_{e}$ in $f_{e}$. With this assumption, the number of REs at the maximum $E_{\gamma}\left(\equiv E_{e}\right)$ is calculated to match the observed $E_{\gamma}$. The full $f_{\gamma}$ expected from this $E_{e}$ is then subtracted from the measured $f_{\gamma}$. This process then repeats working downwards from high to low energy, subtracting the expected $f_{\gamma}$ from progressively lower $E_{e}$, until no residual $f_{\gamma}$ remains. The number of REs needed at each $E_{e}$ to account for the residual $E_{\gamma}$ thus forms $f_{e}$. Since all operations are linear, the calculated $f_{\gamma}$ from the inverted $f_{e}$ always matches the original $f_{\gamma}$.

Uncertainty can also be propagated through this process. Underlying uncertainties are based on Poisson counting-statistics $((\# \gamma) \pm \sqrt{(} \# \gamma))$, and these uncertainties are propagated in quadrature through the onion-peel process. In this way, the absolute $f_{e}$ uncertainties are always larger as $E_{e}$ decreases, but the fractional uncertainty can be significantly smaller if $f_{e}$ increases with decreasing $E_{e}$. To minimize experimental uncertainty, long duration collection times $(\approx 2 \mathrm{~s})$ during stationary periods are used, as well as aggregating pulses collected from multiple spatial views. This increases the raw number of counts and thus reduces the fractional uncertainty.

Whether a given $f_{e}$ is peaked, flat, or inverted depends on the local slope of $f_{\gamma}$. This is shown in Fig. 10, where the inversion results from a family of $f_{\gamma}$ defined by a single slope are shown. For a steep slope in $f_{\gamma}, f_{e}$ is inverted to be peaked, while for shallow $f_{\gamma}, f_{e}$ is inverted to be non-monotonic. This demonstrates that in principle the GRI diagnostic can differentiate between each type of distribution, with $f_{\gamma}$ slope playing a crucial role.

Some assumptions are necessary for the inversion process to be tractable. The first is spatial homogeneity: the same RE phase-space distribution function is assumed to 
exist throughout the sight-line. As discussed in Sec. III. this is less important at high $E_{e}$ since the emission tends to be localized to a smaller volume, but at low $E_{e}$ this is no longer true. Secondly, finite pitch-angle effects are ignored in the inversions, though as discussed in Sec. IIIB further work should enable full 2-D inversions. Alternate inversion processes are also possible, either using weightfunction techniques [45, 46] or tools such as DeGaSum [47, 48.

\section{B. Distribution Function Parametric Dependencies}

The inversion process described above is deployed to compare experimentally reconstructed $f_{e}$ across changes in experimental actuators as well as to compare between modeling and experiment. As discussed in Sec. IIB, changes in electron density $\left(n_{e}\right)$ and toroidal field $\left(B_{T}\right)$ map onto changes of $E / E_{\mathrm{C}}$ (Eq. 1) and $\hat{\tau}_{\text {rad }}$ (Eq. 2) to vary the collisional and synchrotron damping respectively. These non-dimensional parameters are expected from modeling to strongly influence the shape of $f_{e}$.

Results are summarized in Fig. 11. First, considering experimental $f_{e}$ at different $n_{e}$ [different $E / E_{\mathrm{C}}$, Fig. 11(a)], non-monotonicity is observed in the lower energy part of the spectrum. As $n_{e}$ is increased from $1.0 \times 10^{19}$ $\mathrm{m}^{-3}$ to $1.5 \times 10^{19} \mathrm{~m}^{-3}$, large changes in $f_{e}$ are measured well after $t_{\text {puff. }}$ The spectrum is significantly shifted to lower energy and the location of the non-monotonic feature also shifts from $\approx 7$ to $5 \mathrm{MeV}$. Considering modeling (discussed in Sec. IIC) of the same experimental conditions [Fig. 11(b)], agreement is found in some areas. First, the predicted $f_{e}$ contain non-monotonic features at consistent energies to the experimental $f_{e}$. Second, increasing $n_{e}$ shifts $f_{e}$ to lower energy in both modeling and experiment. The main discrepancy is that $f_{e}$ is narrower in experiment than modeling, with $f_{e}$ limited to lower energy. Possible reasons for this discrepancy will be discussed in Sec. VI.

The effect of synchrotron damping is studied by varying $B_{T}$ to affect $\hat{\tau}_{\text {rad }}$, holding $n_{e}$ and $Z$ constant. Looking several seconds after $t_{\text {puff }}$, significant changes in both experimental and modeled $f_{e}$ are found. Experimentally [Fig. 11(c)], opposite effects on $f_{e}$ are observed at high and low $E_{e}$. The largest $B_{T}$ cases are limited to the lowest maximum $E_{e}$, consistent with the maximum energy limit being set by synchrotron damping. An opposite effect is found at low $E_{e}$, where the largest $B_{T}$ case contains the largest low $E_{e}$ population. Since $E / E_{\mathrm{C}}$ is not varying, this is thought to be due to an advective process where at low $B_{T}$ the particles at low $E_{e}$ have simply energized to high $E_{e}$.

Comparing with modeling of the $\hat{\tau}_{\text {rad }}$ effect [Fig. 11(d)], the same qualitative picture is found but with significant quantitative differences. Note first the axis scale is significantly narrower for experimental results, again confirming the narrower nature of the experimental $f_{e}$. The opposite behavior at low and high $E_{e}$ is reproduced, but the 'neutral point' is as $20 \mathrm{MeV}$ in modeling vs. 6 $\mathrm{MeV}$ in experiment. Discussion of these differences will be left to the following section, as the discrepancies are not limited to the energy domain.

To conclude, resolution in the energy domain can be obtained through pulse height analysis to measure $f_{\gamma}$ that in turn can be inverted to $f_{e}$ with some assumptions. This enables comparison of experimental and modeled $f_{e}$ when varying experimental actuators to vary synchrotron and collisional damping. Qualitative agreement is found on the role of these actuators, though quantitative differences are also identified. Measurements are also consistent with predictions of phase-space attractors that accumulate REs and form non-monotonic features in the RE energy distribution.

\section{RESOLVING THE TIME DOMAIN: RE GROWTH AND DECAY}

Slowly varying plasma parameters in the low-density scenario ensures sufficient temporal resolution is available for all diagnostics described in Sec. II A. A complete time-evolution for a well diagnosed discharge is shown in Fig. 12(a)-(e). This discharge included a rise in $B_{T}$ and a relatively small density rise insufficient to bring the emission measured by the distant HXR detector into decay. Very different growth rates are observed across the different diagnostics as summarized in Fig. 12(g). HXR growth rates measured by the GRI system are plotted against the HXR energy $\left(E_{\gamma}\right)$, while other diagnostics are shown as bands owing to their uncertain energy weighting. This indicates the consistency of the GRI growth rates with the energy weighting shown in Fig. 2 2 ECE growth rates are comparable to low $E_{\gamma}$ GRI growth rates while high $E_{\gamma}$ GRI growth rates are comparable to SE. While the energy weighting of the distant HXR is unknown, it is found to generally match the low $E_{\gamma}$ GRI growth rates.

The isolated effects of collisional damping and synchrotron damping will now be considered in Secs. VA and $\mathrm{VB}$. To compare modeled growth rates to the GRI system, a conversion from the time-dependent modeled RE distribution $f_{e}$ to the HXR distribution $f_{\gamma}$ is needed. This is achieved with the GRI synthetic diagnostic described in Sec. III run for many time slices. The timedependence of $f_{e}$ thus maps onto $f_{\gamma}$ time dependence, which is then fit to an exponential function at each $E_{\gamma}$.

\section{A. Effect of Toroidal Field (Synchrotron Damping)}

As toroidal field enters quadratically into $\hat{\tau}_{\text {rad }}$ (Eq. 2), this actuator has a strong effect on emission growth rates as shown in Fig. 13(a). While distant HXR and ECE growth rates increase with increasing $B_{T}$, the $\mathrm{SE}$ growth rate decreases. Qualitatively this can be understood as the low $E_{e}$ RE population decaying more slowly as $B_{T}$ 

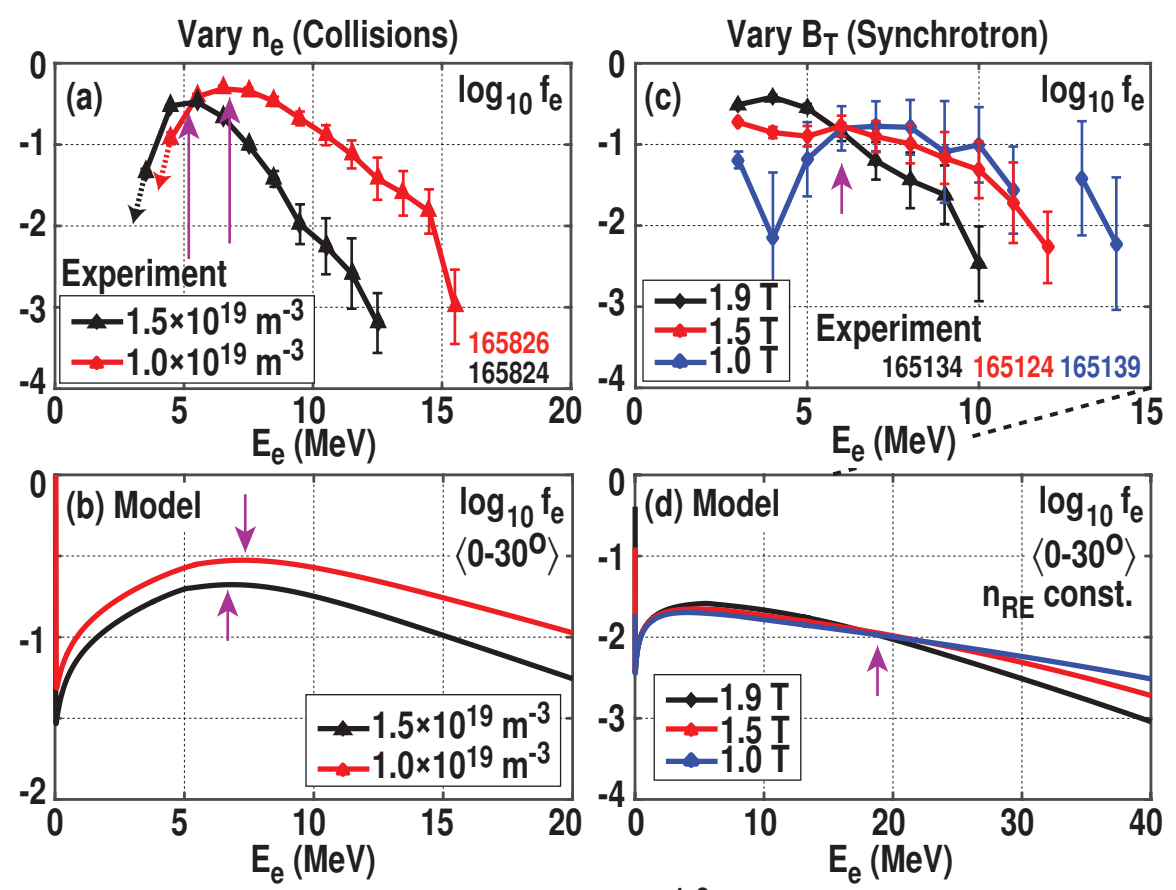

FIG. 11. (a,c) Experimental and (b,d) modeled $f_{e}$ under the effect of $(\mathrm{a}, \mathrm{b}) n_{e}$ and (c,d) $B_{T}$ variations to identify $f_{e}$ sensitivities to $E / E_{\mathrm{C}}$ and $\hat{\tau}_{\text {rad }}$.
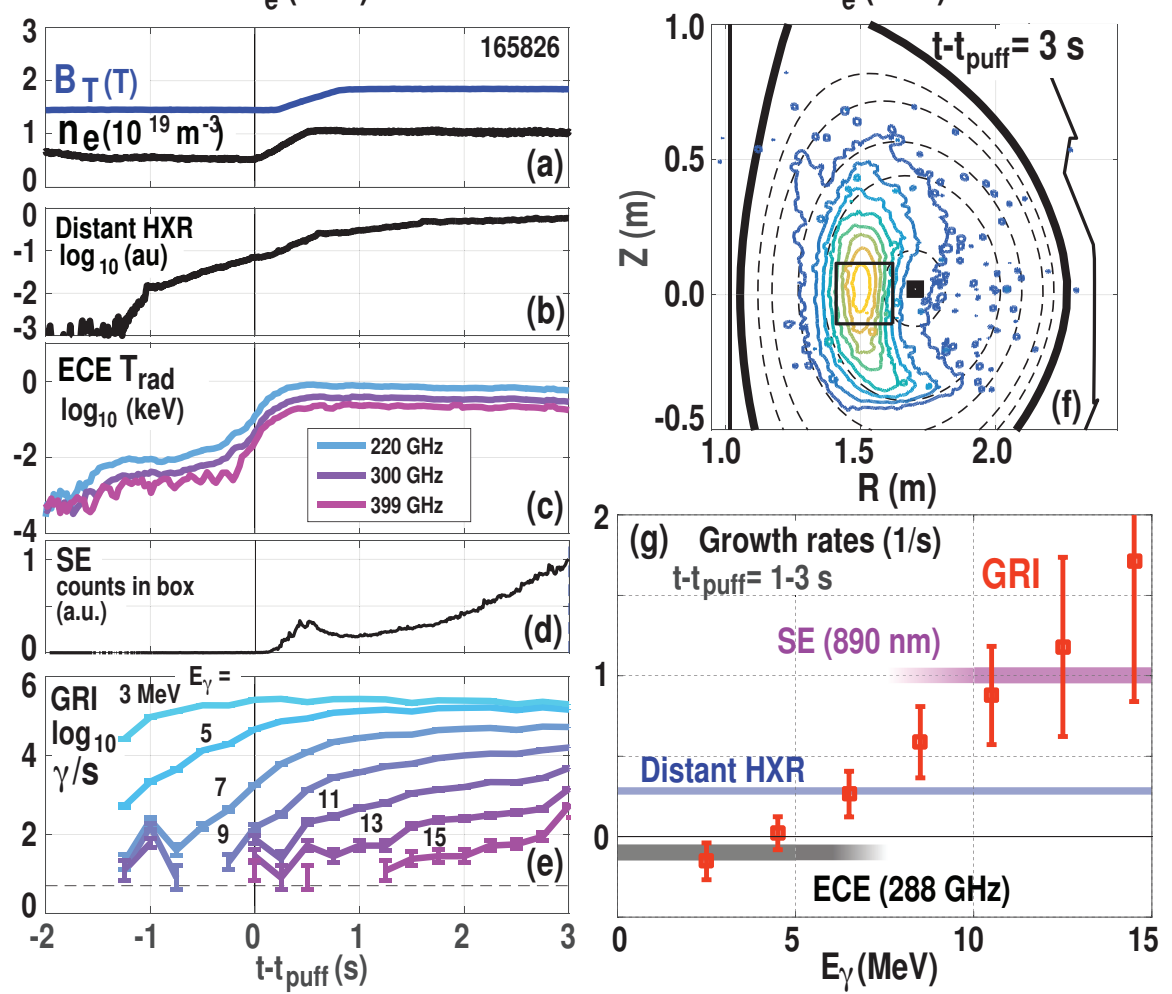

FIG. 12. Time evolution of the discharge across several diagnostics. (a) Increases in $B_{T}$ and $n_{e}$ together after $t_{\text {puff }}$ cause changes in diagnostic growth rates. (b) HXR grows slowly, while (c) ECE is essentially stationary, and (d) SE grows robustly with (f) a HFS-localized emission pattern. Note time-dependence of SE in (d) is obtained by integrated the number of counts in the central box shown in (f). (e) Different GRI growth rates are found at each $E_{\gamma}$, 15 which summarizing in (g) indicates consistency with the other diagnostics.

increases while the highest $E_{e} \mathrm{RE}$ population grows more slowly as $B_{T}$ increases. These measurements are consistent with $E_{\gamma}$-resolved HXR growth rate analysis from the GRI, shown in Fig. 13(b). At low $E_{\gamma}$, the HXR growth rate increases with increasing $B_{T}$ while the high $E_{\gamma}$ growth rate decreases. These $f_{\gamma}$ growth rate changes also demonstrate how the differences in $f_{e}$ with $B_{T}$ shown in Fig. 11(c) arose over time. Initial $f_{e}$ were strongly decaying with $E_{e}$ but over time the low $B_{T}$ case became the most numerous at high $E_{e}$ and least numerous at low $E_{e}$.
Model predictions of the HXR growth rates, shown in Fig. 13 (c), reveal the same qualitative trend. At low $E_{\gamma}$ growth rates increase with $B_{T}$ while the opposite occurs at high $E_{\gamma}$. In modeling the growth rates cross each other at $\approx 8 \mathrm{MeV}$, compared to $5 \mathrm{MeV}$ in experiment. However, the large experimental growth rates shown in Fig. $13(\mathrm{~b})$ with $E_{\gamma}>8 \mathrm{MeV}$ are not recovered until much higher $E_{\gamma}$ in modeling. Note also the different $E_{\gamma}$ axis limits of Fig. 13(b)-(c). Thus the quantitative effect of varying synchrotron damping is found to be larger in 

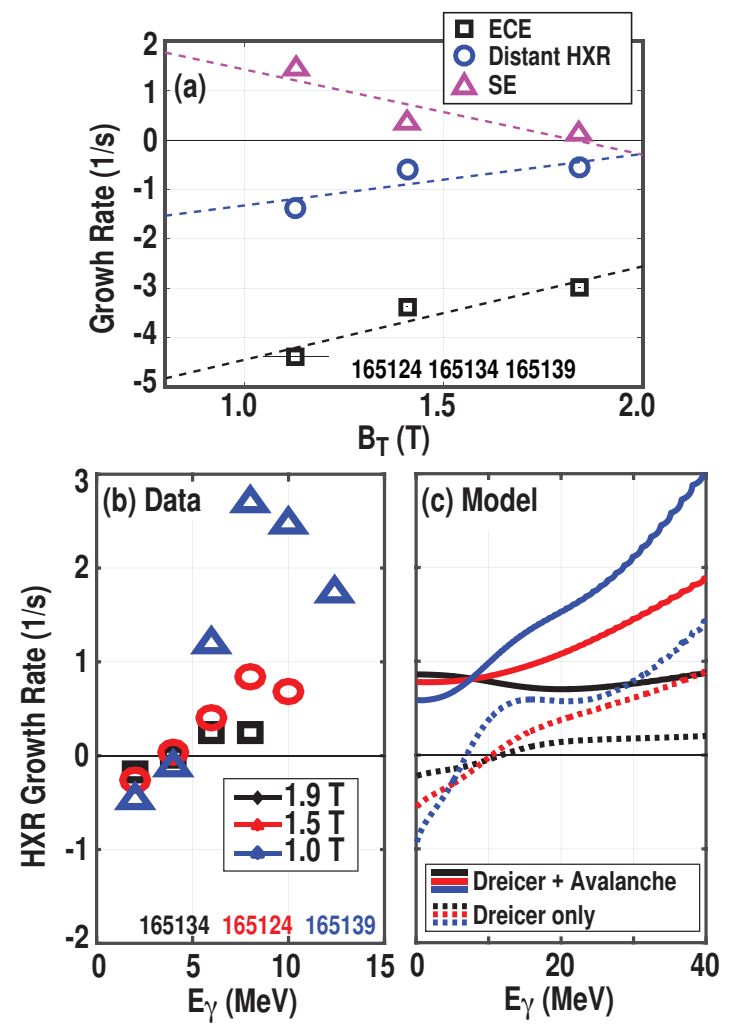

FIG. 13. Dependence of emission growth rates (from 1 to $3 \mathrm{~s}$ after $t_{\text {puff }}$ ) on $B_{T}$ at fixed $n_{e}$ of $2.5 \times 10^{19} \mathrm{~m}^{-3}$ (fixed $E / E_{\mathrm{C}}$ ). (a) Different dependencies are found for diagnostics sensistive to low and high $E_{e}$ populations. (b,c) $E_{\gamma}$-resolved GRI emission growth comparing (b) experiment and (c) modeling.

experiment than modeling.

This discrepancy may be due to the omission of spatial effects in the present modeling, since SE is itself dominated by the HFS region of the plasma [see Fig. 12(f)] where $B$ and thus the local $\hat{\tau}_{\text {rad }}$ is higher. Recent work has also identified the important role of full-orbit spatial effects in enhancing the synchrotron force, with a several-fold increase in stopping power reported 28 .

Focusing on the behavior at low energy $\left(E_{\gamma}<5 \mathrm{MeV}\right)$, a stark qualitative discrepancy is found at all $B_{T}$. While the HXR emission is predicted to grow in modeling, it decays in experiment and thus indicates anomalous $\mathrm{RE}$ loss. Repeating simulations with the avalanche mechanism artificially turned off (dashed lines in Fig. 13(c)) causes the expected signal to decay, as in the experiment. This is only illustrative as in these conditions $\left(E / E_{\mathrm{C}} \approx 4\right)$ avalanching should still occur. A sizeable effect, comparable to the avalanche mechanism, is thus missing from modeling. Possible causes for this will be further described in Sec. VI.

While discharges ramping $B_{T}$ upwards exhibit fairly consistent growth rates in time (as in Fig. 12), ramping $B_{T}$ downwards must be done over very long time scales due to hardware constraints. These discharges thus never reach a stationary condition, and complex temporal dependencies are observed, shown in Fig. 14. While the distant HXR emission and low energy GRI bins are char-
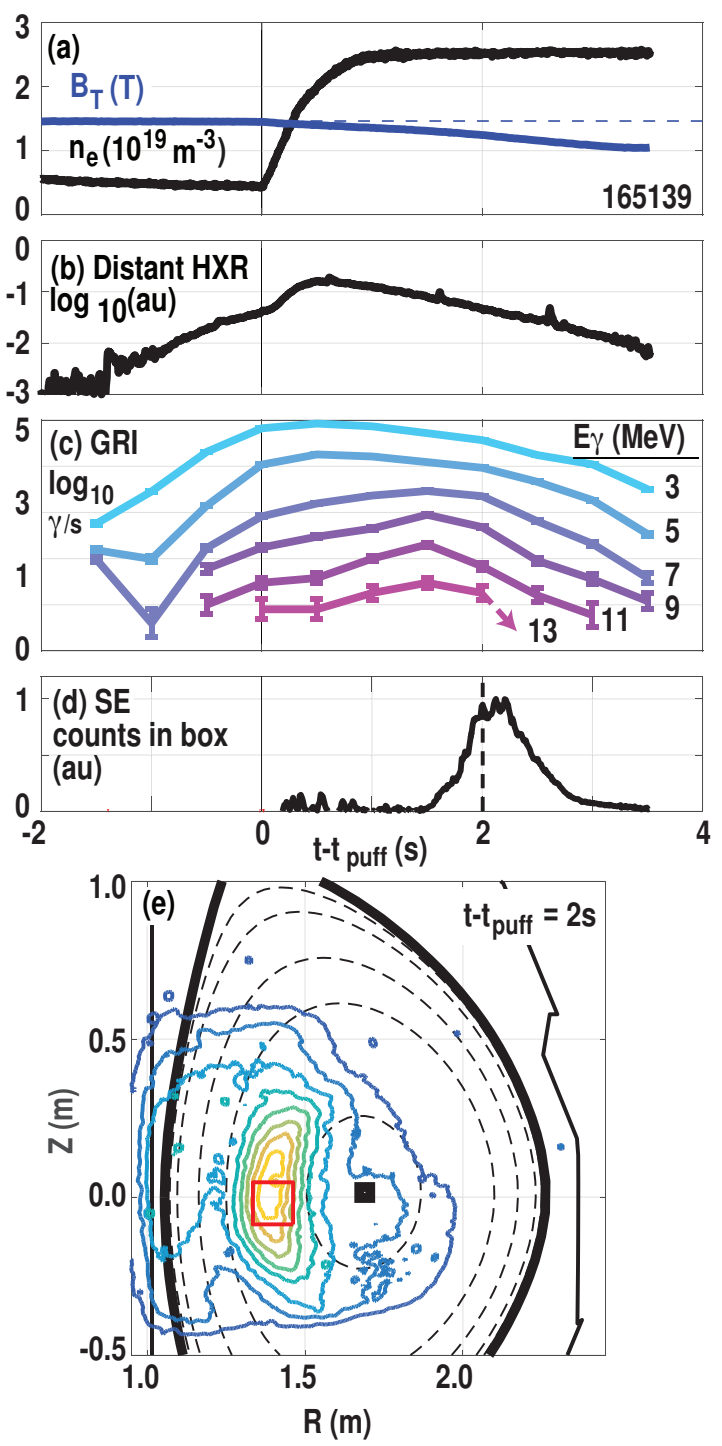

FIG. 14. Complex temporal dependencies for discharges where (a) $B_{T}$ slowly decreases at fixed $n_{e}$. (b) Distant HXR detectors show consistent decay, while (c) high energy GRI and (d) SE show signal growth followed by signal decay. The late decay phase corresponds to (e) increased SE from the region near the center-post, suggesting $\mathrm{RE}$ loss.

acterized by a single decay rate, high energy GRI bins and SE images transition from an early growth phase shortly after $t_{\text {puff }}$ to a decaying phase after $t-t_{\text {puff }}=2 \mathrm{~s}$.

This effect is interpreted as a two-part process. First $f_{e}$ reaches higher peak energy due to the reduction of the synchrotron force. As the REs energize it appears they eventually become deconfined through either a drift orbit effect or some other loss mechanism. This is supported by SE images indicating a region of interaction with the center-post later in time, shown in Fig. 14(e).

To conclude, while the effect of synchrotron damping on RE growth is qualitatively as expected, the comparison shown in Fig. 13 indicates that: 1) the quantitative effect from changing $B_{T}$ is larger than in modeling, and 2) a significant anomaly exists at low $E_{\gamma}$, where HXR 


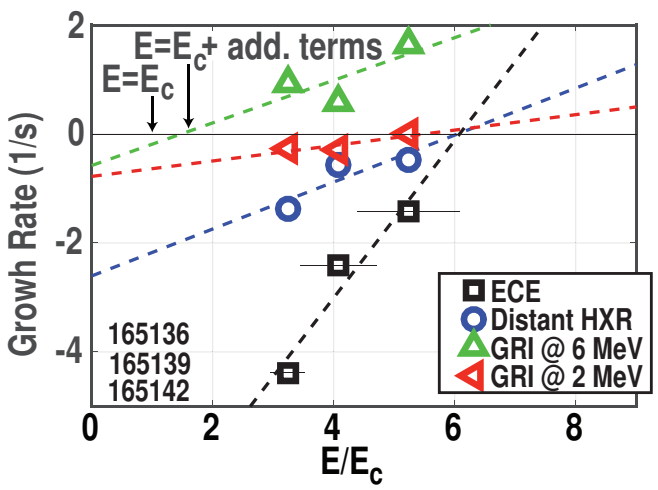

FIG. 15. Dependence of emission growth rates on $E / E_{\mathrm{C}}$, indicating only high $E_{\gamma}$ HXRs extrapolate to a $E / E_{\mathrm{C}}$ zero crossing that is more consistent with the theory in Ref. [9]. Discharges at low $B_{T}$ are presented but findings are similar at other $B_{T}$ values. $Z \approx 1$ for these discharges.
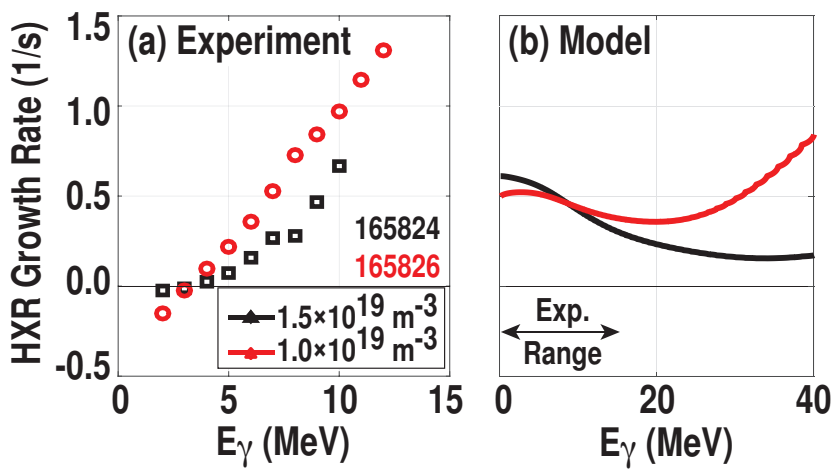

FIG. 16. (a) Experimental and (b) modeled HXR growth rates as a function of HXR energy $\left(E_{\gamma}\right)$ for the cases shown in Fig. 11(a)-(b). $Z \approx 3-4$ for these discharges.

growth is predicted yet decay is observed.

\section{B. Effect of Electron Density (Collisional Damping)}

As the collisional damping rate is proportional to $n_{e}$, Eq. 1 shows $E / E_{\mathrm{C}}$ is inversely proportional to $n_{e}$. Consistent with previous examinations of the $n_{e}$ effect on emission growth rates [18, 19], strong sensitivity to $E / E_{\mathrm{C}}$ is found together with a transition from HXR growth to decay at an anomalously large value of $E / E_{\mathrm{C}}$. Figure 15 demonstrates this result extends to several diagnostics sensitive to low energy REs (ECE, distant HXR, and low energy GRI), but not GRI measurements at high energy. Low energy diagnostics show a transition from emission growth to decay at $E / E_{\mathrm{C}} \approx 6$, which is far in excess of the theoretical expectation even after accounting for expected dissipation enhancement from synchrotron and pitch-angle scattering. These additional dissipation terms raise the expected transition from $E / E_{\mathrm{C}}=1$ to 1.6 in these conditions. In contrast, examining the emission trend from higher energy HXRs measured by the GRI, the extrapolated transition to HXR decay is at an $E / E_{\mathrm{C}}$ value much more consistent with the theory in Ref. 9] (that includes the additional synchrotron and pitch-angle scattering dissipation terms). This suggests the anomalous dissipation of REs is most pronounced at low energy.

Direct comparison of experimental and modeled HXR growth rates as a function of $E_{\gamma}$ is shown in Fig. 16 for two different $n_{e}$ levels. These discharges featured injection of nitrogen to raise $Z$ to between $3-4$, with $n_{e}$ controlled by feedback. Note the lower $n_{e}$ case is also described in Fig. 12. The increased $Z$ increased bremsstrahlung emission and thus signal to noise, enabling more resolved energy-dependent growth rates to be extracted to high energy. Qualitative similarities and differences exist with modeling. Both modeled and experimental growth rates are between 0.5 to $1.51 / \mathrm{s}$, both demonstrate increasing growth rate as $E_{\gamma}$ increases, and both find reduced growth rates as $n_{e}$ increases. However, the horizontal axis is compressed in the experiment, similar to findings shown in Fig. 13. Also consistent with Fig. 13, at low $E_{\gamma}$ HXR growth is expected, yet decaying or flat HXR signals are observed. Interestingly, the very lowest $E_{\gamma}$ growth rates display an opposite dependence to $n_{e}$ as in Fig. 15, presumably due to the presence of high $Z$ in the discharges of Fig. 16. These discrepancies will be further discussed in Sec. VI.

To conclude this final section, time domain resolution is obtained by tracking emission rates as a function of time. Simultaneous growth and decay of different emission bands is observed, and consistency is found between energy-resolved HXR growth rates and other emission bands thought to probe low (ECE) and high (SE) energy REs. Increasing synchrotron damping by raising $B_{T}$ reduces the growth rate of the high $E_{\gamma}$ emission, while increasing $n_{e}$ decreases the growth rate at nearly all energies. Modeling qualitatively recovers these effects, though discrepancies are found as will be discussed in the next section.

\section{DISCUSSION AND CONCLUSION}

In this work diagnostic resolution in the spatial, energy, and temporal domain has yielded novel measurements of RE dependencies to plasma parameters. Broadly speaking, this work has enabled unique opportunities for model validation, and as such improves confidence that modelbased optimization of RE control and avoidance can be achieved. In this section observations are summarized highlighting areas of agreement and disagreement with modeling, followed by discussions of possible resolutions to the identified discrepancies, and finally this work concludes with a discussion of future directions for research.

\section{A. Summary of Observations and Discrepancies with Modeling}

Energy resolution finds qualitative agreement of distribution function changes with collisional and synchrotron 
damping: increased collisional damping reduces the energy of $f_{e}$ throughout, while increased synchrotron damping reduces the high-energy part of $f_{e}$. Many $f_{e}$ are found to develop non-monotonic features as the discharge develops, and the energy of these features are consistently predicted by modeling. Consistently across all conditions, experimentally measured $f_{e}$ appear 'narrower' than predicted, with $f_{e}$ decreasing with energy faster in experiment. Time resolution reveals that considering the HXR growth rate as a function of energy yields consistent (and with improved resolution) growth rates to other diagnostics. Quantitative discrepancies with predicted growth rates are found for all energies, but the most severe qualitative discrepancy is found at low energy. Decay of low energy HXRs are observed, for $E / E_{\mathrm{C}}$ up to $\approx 5$, while growth is predicted for $E / E_{\mathrm{C}}>1.6$. Consistently, spatial resolution identifies radial gradients of the RE population, with the lowest gradients (and thus presumably the largest transport) found at low energy. Possible resolutions to these discrepancies are now described.

\section{B. Possible Resolutions to Discrepancies}

Modeling utilized to describe this work takes into account the physical mechanisms thought to be important for the RE distribution function, such as Dreicer generation, avalanche multiplication, collisional damping, synchrotron damping, and pitch-angle scattering. However, potentially important aspects are still missing, the most important of which are proposed here to be spatial transport and the role of kinetic instability.

As described in Sec. III A, radial gradients are found in the RE population. These gradients could be related to a core source and radial diffusive flux term, by experimentally fitting a diffusion coefficient that matches the data (varying with energy). However, modeling at present is unable to predict the RE diffusion from first principles without knowing the magnitude and spectrum of the fluctuating magnetic field components. Thus it is difficult to quantify the role of spatial diffusion, though admittedly the fact that gradients are weakest at low energy is contrary to simple expectations for fluctuation-based RE transport. Future experiments may explore the role of spatial diffusion more carefully by varying the magnitude of the thermal confinement and also by exploring effects due to imposed non-axisymmetric magnetic perturbations.

A second possibility is kinetic instability of the RE beam itself. This would manifest itself as waves driven by the RE population through either the Cerenkov, normal Doppler, or anomalous Doppler resonances. A wide spectrum of waves can be driven by the RE population, and indeed the non-monotonic $f_{e}$ found in these studies should have a strong drive for kinetic instability. Recent work has identified a strong drive for magneto-sonic waves at $10 \mathrm{~s}$ of $\mathrm{GHz}$ from these $f_{e}$ 49, and interestingly the resonances leading to the strongest RE diffu- sion are found at low $E_{e}$, where the largest discrepancy with modeling is found. Future dedicated work will thus focus on the potential role of kinetic instability to explain the discrepancies found in these studies. It should however be noted that the keV-level $T_{e}$ Ohmic plasmas used for this study are rather collisionless and thus only weakly damp kinetic instabilities. In contrast, the much colder and collisional conditions (eV-level $T_{e}$ ) of the postdisruption ITER RE beam suggests it is should be significantly harder for kinetic instability to play a role in post-disruption RE dissipation [50].

\section{Future Directions}

Beyond the model improvements described in Sec. VIB future experimental and analysis work is planned along several fronts. Firstly, measurements here focus on the low-density RE regime. At present, measurements in the post-disruptive RE regime on DIII-D [3] have been limited due to the much higher HXR fluxes saturating the GRI diagnostic. Increased shielding and improved electronics are planned to reduce the stray HXR flux and also increase the maximum count rate. Further, measurements here included only a subset of the available 'pixels' due to a limited number of detectors. Planned upgrades will double the number of active channels to fully exploit the spatial resolution of the GRI diagnostic. Considering inversions of $f_{\gamma}$ to $f_{e}$, work is planned to include finite pitch-angle effects in the synthetic diagnostic used to generate the HXR spectra shown in Fig. 9. Utilizing multiple views of the same flux surface to gain pitch-angle resolution should allow a full energy and pitch-angle resolved inversion of the RE distribution function.

\section{ACKNOWLEDGMENTS}

DIII-D data shown in this paper can be obtained in digital format by following the links at https:// fusion.gat.com/global/D3D_DMP. The authors thank J. Kulchar, D. Taussig, M. Austin, S. Haskey, B. Grierson, R. Groebner, and Y. Zhu for diagnostic support, as well as N. Commaux and A. Wingen for their assistance. This material is based upon work supported in part by the U.S. Department of Energy under Grants DE-FC02-04ER54698, DE-FG02-07ER54917, DE-AC0500OR22725, DE-FC02-99ER54512, DE-SC0016268.

DISCLAIMER: This report was prepared as an account of work sponsored by an agency of the United States Government. Neither the United States Government nor any agency thereof, nor any of their employees, makes any warranty, express or implied, or assumes any legal liability or responsibility for the accuracy, completeness, or usefulness of any information, apparatus, product, or process disclosed, or represents that its use would not infringe privately owned rights. Reference herein to any specific commercial product, process, or service by 
trade name, trademark, manufacturer, or otherwise, does not necessarily constitute or imply its endorsement, recommendation, or favoring by the United States Government or any agency thereof. The views and opinions of authors expressed herein do not necessarily state or reflect those of the United States Government or any agency thereof.
[1] T. C. Hender, J. C. Wesley, J. M. Bialek, A. Bondeson, A. H. Boozer, R. J. Buttery, A. M. Garofalo, T. P. Goodman, R. S. Granetz, Y. Gribov, O. Gruber, M. P. Gryaznevich, G. Giruzzi, S. Günter, N. Hayashi, P. Helander, C. C. Hegna, D. F. Howell, D. A. Humphreys, G. T. A. Huysmans, A. W. Hyatt, A. Isayama, S. C. Jardin, Y. Kawano, A. G. Kellman, C. E. Kessel, H. R. Koslowski, R. J. LaHaye, E. Lazzaro, Y. Liu, V. Lukash, J. Manickam, S. Medvedev, V. Mertens, S. V. Mirnov, Y. Nakamura, G. A. Navratil, M. Okabayashi, T. Ozeki, R. Paccagnella, G. Pautasso, F. Porcelli, V. D. Pustovitov, V. Riccardo, M. Sato, O. Sauter, M. J. Schaffer, M. Shimada, P. Sonato, E. J. Strait, M. Sugihara, M. Takechi, A. D. Turnbull, E. Westerhof, D. G. Whyte, R. Yoshino, H. Zohm, D. Group, the Itpa Mhd, and Magnet, Nuclear Fusion 47, S128 (2007)

[2] M. Lehnen, K. Aleynikova, P. B. Aleynikov, D. J. Campbell, P. Drewelow, N. W. Eidietis, Y. Gasparyan, R. S. Granetz, Y. Gribov, N. Hartmann, E. M. Hollmann, V. A. Izzo, S. Jachmich, S.-H. Kim, M. Kočan, H. R. Koslowski, D. Kovalenko, U. Kruezi, A. Loarte, S. Maruyama, G. F. Matthews, P. B. Parks, G. Pautasso, R. A. Pitts, C. Reux, V. Riccardo, R. Roccella, J. A. Snipes, A. J. Thornton, and P. C. de Vries, Journal of Nuclear Materials 463, 39 (2015).

[3] E. M. Hollmann, P. B. Aleynikov, T. Fülöp, D. A. Humphreys, V. A. Izzo, M. Lehnen, V. E. Lukash, G. Papp, G. Pautasso, F. Saint-Laurent, and J. A. Snipes, Physics of Plasmas 22, 021802 (2015).

[4] A. H. Boozer, Physics of Plasmas 22 (2015), 10.1063/1.4913582

[5] J. W. Connor and R. J. Hastie, Nuclear Fusion 15, 415 (1975)

[6] M. N. Rosenbluth and S. V. Putvinski, Nuclear Fusion 37, 1355 (1997)

[7] J. R. Martin-Solis, J. D. Alvarez, R. Sanchez, and B. Esposito, Physics of Plasmas 5, 2370 (1998).

[8] F. Andersson, P. Helander, and L.-G. Eriksson, Physics of Plasmas 8, 5221 (2001)

[9] P. B. Aleynikov and B. N. Breizman, Physical Review Letters 114, 155001 (2015)

[10] P. B. Aleynikov, K. Aleynikova, B. N. Breizman, G. T. A. Huijsmans, S. V. Konovalov, S. V. Putvinski, and V. Zhogolev, in Proc. of 25th IAEA Fusion Energy Conf. (St. Petersburg, Russia) (2014) pp. TH/P3-38.

[11] E. Hirvijoki, I. Pusztai, J. Decker, O. Embréus, A. Stahl, and T. Fülöp, Journal of Plasma Physics 81, 475810502 (2015), arXiv:1502.03333

[12] A. Stahl, E. Hirvijoki, J. Decker, O. Embréus, and T. Fülöp, Phys. Rev. Lett. 114, 115002 (2015)

[13] C. Liu, D. P. Brennan, A. H. Boozer, and A. Bhattacharjee, Physics of Plasmas 23, 1 (2016), arXiv:1509.04402

[14] J. Decker, E. Hirvijoki, O. Embréus, Y. Peysson, A. Stahl, I. Pusztai, and T. Fülöp, Plasma Physics and Controlled Fusion 58, 025016 (2016)
[15] C. Liu, D. P. Brennan, A. H. Boozer, and A. Bhattacharjee, Plasma Physics and Controlled Fusion 59, 024003 (2017).

[16] Z. Guo, C. J. Mcdevitt, and X. Tang, Plasma Physics and Controlled Fusion 59, 044003 (2017).

[17] J. R. Martin-Solis, R. Sánchez, and B. Esposito, Physical Review Letters 105, 185002 (2010)

[18] C. Paz-Soldan, N. W. Eidietis, R. S. Granetz, E. M. Hollmann, R. A. Moyer, N. A. Crocker, A. Wingen, and Y. Zhu, Physics of Plasmas 21, 022514 (2014).

[19] R. S. Granetz, B. Esposito, J. H. Kim, R. Koslowski, M. Lehnen, J. R. Martin-Solis, C. Paz-Soldan, T. Rhee, J. C. Wesley, L. Zeng, and I. M. Group, Physics of Plasmas 21, 072506 (2014).

[20] E. M. Hollmann, P. B. Parks, D. A. Humphreys, N. H. Brooks, N. Commaux, N. W. Eidietis, T. E. Evans, R. Isler, A. N. James, T. C. Jernigan, J. Munoz, E. J. Strait, C. Tsui, J. C. Wesley, and J. H. Yu, Nuclear Fusion 51, 103026 (2011)

[21] E. M. Hollmann, P. B. Parks, N. Commaux, N. W. Eidietis, R. A. Moyer, D. Shiraki, M. E. Austin, C. J. Lasnier, C. Paz-Soldan, and D. L. Rudakov, Physics of Plasmas 22, 056108 (2015).

[22] C. Paz-Soldan, C. M. Cooper, P. B. Aleynikov, D. C. Pace, N. W. Eidietis, D. P. Brennan, R. S. Granetz, E. M. Hollmann, C. Liu, A. Lvovskiy, R. A. Moyer, and D. Shiraki, Physical Review Letters 118, 255002 (2017).

[23] D. C. Pace, C. M. Cooper, D. Taussig, N. W. Eidietis, E. M. Hollmann, V. Riso, and M. A. V. Zeeland, Review of Scientific Instruments 87, 043507 (2016).

[24] C. M. Cooper, D. C. Pace, C. Paz-Soldan, N. Commaux, N. W. Eidietis, E. M. Hollmann, and D. Shiraki, Review of Scientific Instruments 87, 11E602 (2016)

[25] R. Jaspers, N. Cardozo, and K. H. Finken, Physical Review Letters 72, 4093 (1994).

[26] J. H. Yu, E. M. Hollmann, N. Commaux, N. W. Eidietis, D. A. Humphreys, A. N. James, T. C. Jernigan, and R. A. Moyer, Physics of Plasmas 20, 042113 (2013)

[27] R. J. Zhou, I. M. Pankratov, L. Q. Hu, M. Xu, and J. H. Yang, Physics of Plasmas 21, 063302 (2014).

[28] L. Carbajal, D. Del-Castillo-Negrete, D. Spong, S. Seal, and L. R. Baylor, Physics of Plasmas 24, 042512 (2017)

[29] M. Hoppe, O. Embréus, R. A. Tinguely, R. S. Granetz, A. Stahl, and T. Fülöp, (2017) arXiv:1709.00674.

[30] H. Dreicer, Phys. Rev. 115, 238 (1959)

[31] C. Paz-Soldan, R. J. La Haye, D. Shiraki, R. J. Buttery, N. W. Eidietis, E. M. Hollmann, R. A. Moyer, J. E. Boom, and I. T. Chapman, Nuclear Fusion 56, 056010 (2016)

[32] L.-G. Eriksson and P. Helander, Computer Physics Communications 154, 175 (2003).

[33] P. Helander, Collisional Transport in Magnetized Plasmas (Cambridge University Press, 2002).

[34] G. Papp, M. Drevlak, T. Fülöp, and P. Helander, $\mathrm{Nu}-$ clear Fusion 51, 43004 (2011) 
35] P. B. Parks, M. N. Rosenbluth, and S. Putvinski, Physics of Plasmas 6, 2523 (1999)

[36] P. B. Aleynikov and B. N. Breizman, Nuclear Fusion 57, 046009 (2017)

[37] J. R. Martin-Solis, A. Loarte, and M. Lehnen, Nuclear Fusion 57, 066025 (2017)

[38] V. Zhogolev and S. V. Konovalov, VANT series Nuclear Fusion 4 (2014).

[39] L. Hesslow, O. Embréus, A. Stahl, T. C. Dubois, G. Papp, S. L. Newton, and T. Fülöp, Physical Review Letters 118, 1 (2017), arXiv:1705.08638.

40] E. M. Hollmann, N. Commaux, N. W. Eidietis, C. J. Lasnier, D. L. Rudakov, D. Shiraki, C. Cooper, J. R. Martin-Solis, P. B. Parks, and C. Paz-Soldan, Physics of Plasmas 24, 062505 (2017).

[41] C. Reux, V. Plyusnin, B. Alper, D. Alves, B. Bazylev, and E. Belonohy, Nuclear Fusion 55, 093013 (2015)

[42] P. Helander, L.-G. Eriksson, and F. Andersson, Physics of Plasmas 7, 4106 (2000)

[43] H. W. Koch and J. W. Motz, Review of Modern Physics 31, 920 (1959)
[44] Y. Peysson and F. Imbeaux, Review of Scientific Instruments 70, 3987 (1999).

[45] M. Salewski, M. Nocente, G. Gorini, A. Jacobsen, V. Kiptily, S. Korsholm, F. Leipold, J. Madsen, D. Moseev, S. Nielsen, J. Rasmussen, M. Stejner, and M. Tardocchi, Nuclear Fusion 56, 046009 (2016)

[46] L. Stagner and W. W. Heidbrink, Physics of Plasmas 24 (2017), 10.1063/1.4990391.

[47] A. E. Shevelev, E. M. Khilkevitch, V. G. Kiptily, I. N. Chugunov, D. B. Gin, D. N. Doinikov, V. O. Naidenov, A. E. Litvinov, and I. A. Polunovskii, Nuclear Fusion 53, 123004 (2013).

[48] A. E. Shevelev, E. M. Khilkevitch, S. I. Lashkul, V. V. Rozhdestvensky, and A. B. Altukhov, Nuclear Instruments and Methods in Physics Research A 830, 102 (2016).

[49] C. Liu, E. Hirvijoki, G.-y. Fu, D. P. Brennan, A. Bhattacharjee, and C. Paz-Soldan, ArXiv e-prints (2018)

[50] P. B. Aleynikov and B. N. Breizman, Nuclear Fusion 55, 043014 (2015) 